\title{
ZnO Catalyzed Efficient Synthesis of Some New 2-Substituted-4,6-diarylpyrimidines
}

\author{
K. L. Ameta, Biresh Kumar, and Nitu S. Rathore \\ Department of Chemistry, Faculty of Arts, Science and Commerce, Mody Institute of Technology \& Science, \\ Lakshmangarh 332311, Rajasthan, India
}

Correspondence should be addressed to K. L. Ameta, klameta77@yahoo.co.in

Received 5 September 2012; Accepted 24 September 2012

Academic Editors: G. Gattuso, J. Ishihara, J. L. Jios, and J.-P. Praly

Copyright ( $) 2012$ K. L. Ameta et al. This is an open access article distributed under the Creative Commons Attribution License, which permits unrestricted use, distribution, and reproduction in any medium, provided the original work is properly cited.

A simple and efficient protocol is developed for the synthesis of 2-substituted-4,6-diarylpyrimidines from one-pot threecomponent reaction of $4^{\prime}$-hydroxy-3', $5^{\prime}$-dinitro substituted chalcones, S-benzylthiouronium chloride (SBT), and heterocyclic secondary amines (morpholine/pyrrolidine/piperidine) in the presence of $15 \mathrm{~mol} \% \mathrm{of} \mathrm{ZnO}$ as a heterogeneous catalyst. The present methodology offers several advantages such as being a simple procedure as well as providing excellent yields, and short reaction time. The catalyst is inexpensive, stable, and can be easily recycled and reused for several cycles with consistent activity.

\section{Introduction}

Since many decades, bioactive heterocyclic compounds are one of the main topics of interest for the medicinal chemists as it displays a number of pharmacological activities. Nitrogen, sulphur, and oxygen containing five- and sixmembered heterocyclic compounds have occupied enormous significance in the field of medicinal chemistry. The multicomponent reactions (MCRs) have emerged as an efficient and powerful tool in modern synthetic organic chemistry allowing the facile creation of several new bonds in a one-pot reaction. Therefore, in the last decade, research in academia and industry has increasingly emphasized the use of MCRs as well as domino reaction sequences for a broad range of products $[1,2]$. Due to the atom economy, convergent character, and simplicity of one-pot procedures, multicomponent condensation reactions (MCRs) have an advantageous position among other reactions. The discovery and development of novel MCRs is receiving a growing interest from industrial chemistry research groups and represents a new challenge for organic chemists and to the basic understanding of organic chemistry itself [3].

Recently, intensive studies have been focused on the development of catalytic systems owing to their importance in synthetic organic chemistry. One of the most attractive synthetic strategies favoured by organic chemists is the use of heterogeneous catalyst in increasing the efficiency of a wide range of organic synthesis. Heterogeneous catalysis is being used in the fine chemical industries because of the need for more environmental friendly production technology. This tendency is assisted by the availability of catalytic materials and modern techniques of creating and investigating specific active sites on catalyst surfaces $[4,5]$. Metal oxides exhibit both Lewis acid and Lewis base character at their surface [6].

The basic skeleton of chalcones possesses an $\alpha, \beta$ unsaturated carbonyl group, used as the starting material for the synthesis of variously substituted different sized heterocycles of physiological importance like antiviral [7], antimalarial [8-10], antileishmanial [11, 12], antioxidant [13], anticancer $[14,15]$, and antimicrobial [16]. Pyrimidines and its derivatives are known as an important class of heterocyclic compounds in the pharmaceutical industry as well as in synthetic Chemistry [17]. On the other hand, the pyrimidines unit is a privileged heterocyclic motif that forms the core of a large family of nucleic acids and natural products with strong bioactivity profiles and significant structural properties [18].

Keeping in view of diverse biological activities of pyrimidines, it was thought to construct a novel system which may combine these bioactive rings together in a single 
molecular framework to see the additive effects towards their biological activities. Hence, as a part of our ongoing program to develop efficient and robust methods for the preparation of biologically relevant compounds [19], we have developed a facile and efficient catalytic approach for the multicomponent one-pot synthesis of novel 2substituted-4,6-diarylpyrimidines (Scheme 1).

S-benzylthiouronium chloride (SBT) [20] and dicyandiamide (DDA) [21] have emerged from our laboratory team as versatile reagents for the continued synthesis of 2, 4, 6-tri substituted pyrimidines from $\alpha, \beta$-unsaturated ketones and heterocyclic secondary amines. Consequently, facile synthesis of substituted pyrimidines was carried out using SBT, $\alpha$, $\beta$-unsaturated ketones and organic bases under microwave irradiation with [22] or without [23] solvents.

To the best of our knowledge, there is no report available in the literature describing the use of $\mathrm{ZnO}$ as a catalyst for the synthesis of pyrimidine derivatives. $\mathrm{ZnO}$ is very reactive because it offers higher surface area and low coordinating sites. The surface area of the catalyst increases tremendously when size decreases and is responsible for the higher catalytic activity [24]. The effectiveness of the process was studied by comparing the results obtained with and without catalyst under normal conditions. Herein, we wish to report a novel synthesis of 4,6-diaryl-2-(4-morpholinyl/1-pyrrolidinyl/1piperidinyl)-pyrimidines using $\mathrm{ZnO}$ as an efficient, nontoxic, reusable, and commercially available catalyst.

\section{Results and Discussion}

As a part of our ongoing research interest aimed at developing new synthetic strategies for the bioactive heterocyclic framework, the reaction of chalcones, S-benzylthiouronium chloride, and heterocyclic secondary amines was examined in the presence of catalytic amount $(15 \mathrm{~mol} \%)$ of $\mathrm{ZnO}$ under stirring condition at $100^{\circ} \mathrm{C}$ for 6 hour to give 2substituted-4,6-diarylpyrimidine derivatives up to quantitative yields. A conceivable mechanism for the formation of the product would be as follows: the $\mathrm{ZnO}$ particle facilitates the Michael addition type coupling through Lewis acid sites $\left(\mathrm{Zn}^{2+}\right)$ coordinated to the enone functionality. On the other hand, $\mathrm{ZnO}$ particles can activate SBT so that deprotonation of the $\mathrm{N}-\mathrm{H}$ bond occurs in the presence of Lewis basic sites $\left(\mathrm{O}^{2-}\right)$. As a result, the formation of 4,6-diaryl-2-benzylthiopyrimidines proceeds by activation of reactants through both Lewis acids and basic sites of $\mathrm{ZnO}$ catalyst and then nucleophilic substitution occurs by heterocyclic secondary amines and afforded 4,6-diaryl-2-(4morpholinyl/1-pyrrolidinyl/1-piperidinyl)-pyrimidines. The catalyst could be recovered easily by solvent extraction of the product from the reaction mixture. For this, ethyl acetate was used; the aqueous layer containing the $\mathrm{ZnO}$ particles could be used for the next cycle.

The reaction was optimized for various reaction parameters such as temperature, solvent, and catalyst loading. The chalcones remain unconsumed when the reaction was done at room temperature. The effect of temperature on the yield of product was monitored from 60 to $120^{\circ} \mathrm{C}$ (Table 1, entry
TABLE 1: Effect of temperature for the synthesis of 2-substituted4,6-diarylpyrimidine ${ }^{\mathrm{a}}$.

\begin{tabular}{lcc}
\hline Entry & Temperature $\left({ }^{\circ} \mathrm{C}\right)$ & Yield $(\%)$ \\
\hline 1 & Room temp & Nil \\
2 & 60 & 30 \\
3 & 80 & 60 \\
4 & 100 & 90 \\
5 & 120 & 90 \\
\hline
\end{tabular}

${ }^{a}$ Reaction conditions: chalcone $(0.002 \mathrm{~mol})$, SBT $(0.0022 \mathrm{~mol})$, heterocyclic secondary amine $(0.0024 \mathrm{~mol})$, and $\mathrm{ZnO}$ catalyst $(15 \mathrm{~mol} \%)$ in $\mathrm{DMF}$ $(10 \mathrm{~mL})$ at $100^{\circ} \mathrm{C}$ temperature for $6 \mathrm{~h}$.

TABLE 2: Effect of solvent for the synthesis of 2-substituted-4,6diarylpyrimidine ${ }^{\mathrm{a}}$.

\begin{tabular}{lcc}
\hline Entry & Solvent & Yield (\%) \\
\hline 1 & None & 20 \\
2 & $\mathrm{H}_{2} \mathrm{O}$ & 30 \\
3 & $\mathrm{NMP}$ & 68 \\
4 & $\mathrm{DMSO}$ & 75 \\
5 & $\mathrm{DMF}$ & 90 \\
\hline
\end{tabular}

${ }^{a}$ Reaction conditions: chalcone $(0.002 \mathrm{~mol})$, SBT $(0.0022 \mathrm{~mol})$, heterocyclic secondary amine $(0.0024 \mathrm{~mol})$, and $\mathrm{ZnO}$ catalyst $(15 \mathrm{~mol} \%)$ in DMF $(10 \mathrm{~mL})$ at $100^{\circ} \mathrm{C}$ temperature for $6 \mathrm{~h}$.

TABLE 3: Effect of catalyst loading for the synthesis of 2-substituted4,6-diarylpyrimidine ${ }^{\mathrm{a}}$.

\begin{tabular}{lcc}
\hline Entry & Catalyst $(\mathrm{mol} \%)$ & Yield $(\%)$ \\
\hline 1 & 5 & 45 \\
2 & 10 & 60 \\
3 & 15 & 90 \\
4 & 20 & 90 \\
\hline
\end{tabular}

${ }^{a}$ Reaction conditions: chalcone $(0.002 \mathrm{~mol})$, SBT $(0.0022 \mathrm{~mol})$, heterocyclic secondary amine $(0.0024 \mathrm{~mol})$, and $\mathrm{ZnO}$ catalyst in $\mathrm{DMF}(10 \mathrm{~mL})$ at $100^{\circ} \mathrm{C}$ temperature for $6 \mathrm{~h}$.

3b). However, no further increase in the yield was obtained by increasing the temperature from 100 to $120^{\circ} \mathrm{C}$. Hence $100^{\circ} \mathrm{C}$ was chosen as optimum reaction temperature.

An attempt to catalyze the reaction in the absence of solvent resulted in very low yields (Table 2, entry $\mathbf{3 b}$ ). Among the various solvents studied, DMF was found to be the best solvent giving maximum yield of desired product.

Catalyst concentration was optimized by varying its concentration from 5 to $20 \mathrm{~mol} \%$ (Table 3, entry $3 \mathbf{b}$ ). Increase in the product yield was observed form 5 to $20 \mathrm{~mol} \%$ of catalyst amount. Hence $15 \mathrm{~mol} \%$ was considered as an optimum catalyst concentration. The transformations of the reacting species were also confirmed by the spectroscopic studies. In FTIR, the disappearance of band at $1680-1710 \mathrm{~cm}^{-1}$ was due to the carbonyl group of chalcone, the appearance of band at $1595-1630 \mathrm{~cm}^{-1}$ due to cyclization, and in ${ }^{1} \mathrm{H}$ NMR the appearance of multiplet at $\delta 3.40-3.90$ for the $-\mathrm{CH}_{2}-\mathrm{N}-$ $\mathrm{CH}_{2}$ - of morpholine/pyrrolidine/piperidine. 

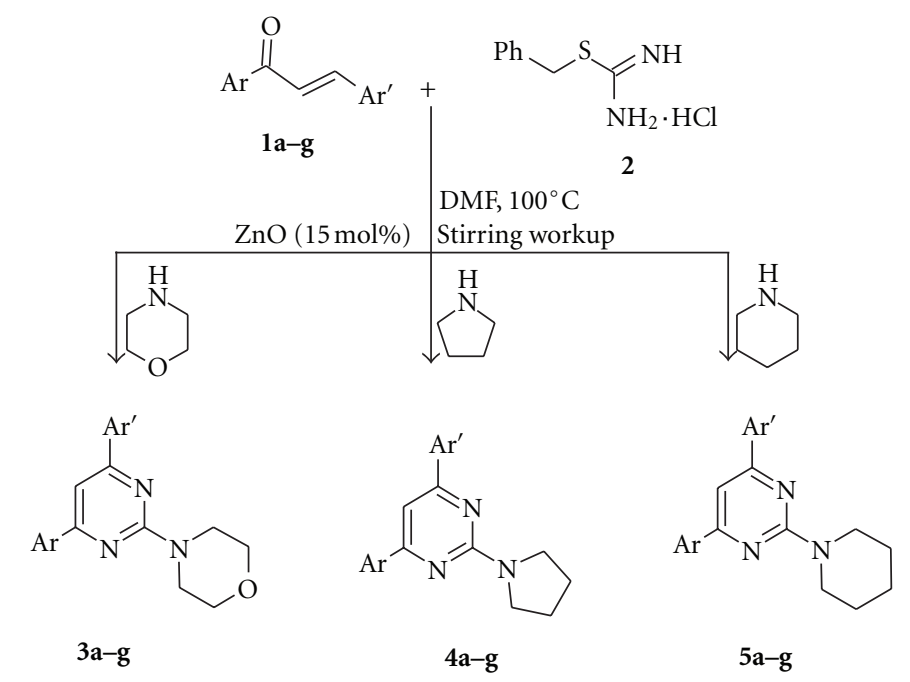

Ar: 4-(OH)-3,5- $\left(\mathrm{NO}_{2}\right)_{2}-\mathrm{C}_{6} \mathrm{H}_{2}$

$\mathrm{Ar}^{\prime}$ : (a) $-\mathrm{C}_{6} \mathrm{H}_{5}$, (b) $2-\mathrm{Br}^{-} \mathrm{C}_{6} \mathrm{H}_{4}$, (c) $4-\mathrm{Br}_{-}-\mathrm{C}_{6} \mathrm{H}_{4}$, (d) $2-\mathrm{Cl}_{-} \mathrm{C}_{6} \mathrm{H}_{4}$,

(e) $4-\mathrm{OH}-\mathrm{C}_{6} \mathrm{H}_{4}$, , f $) 4-\mathrm{Cl}-\mathrm{C}_{6} \mathrm{H}_{4},\left(\right.$ g) $3-\mathrm{Cl}-\mathrm{C}_{6} \mathrm{H}_{4}$

Scheme 1: Synthesis of the title compounds.

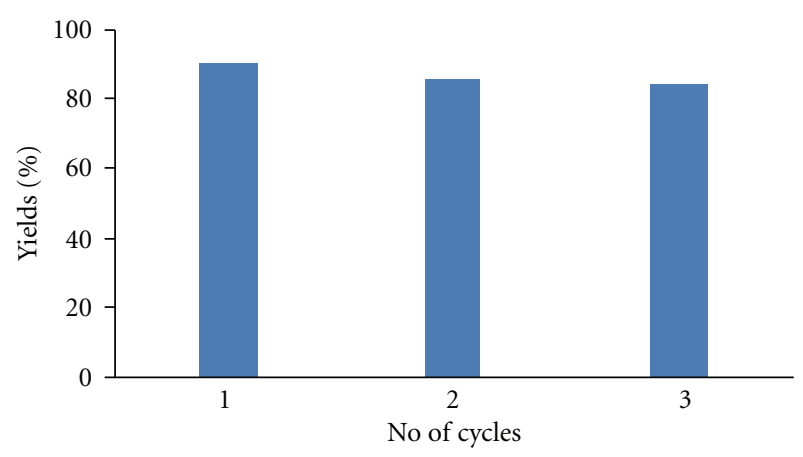

FIgURE 1: Recyclability of $\mathrm{ZnO}$ catalyst.

Reusability is one of the important properties of this catalyst. In this study, the catalyst was recovered by filtration from the reaction mixture and reused during three consecutive runs without any apparent loss of activity for the same reaction Figure 1. In order to confirm the effective involvement of $\mathrm{ZnO}$ catalyst during transformation, we also performed the experiment under conventional heating without using catalyst [25].

\section{Conclusion}

We have developed a simple, economic, eco-friendly and highly efficient synthetic strategy for exclusive synthesis of 2-substituted-4,6-diarylpyrimidines using inexpensive, recyclable, and commercially available catalyst. On the other hand, $\mathrm{ZnO}$ is remarkably easier to use, nonhazardous, inexpensive, and works under mild conditions.

\section{Experimental Section}

4.1. General. The reaction mixtures were stirred magnetically. Chemicals were purchased from Sigma-Aldrich, Merck, and used without further purification. ${ }^{1} \mathrm{H}$ - and ${ }^{13} \mathrm{C} \mathrm{NMR}$ spectra were recorded using $\left(\mathrm{CDCl}_{3}\right)$ on $400 \mathrm{MHz}{ }^{1} \mathrm{H} \mathrm{NMR}$ spectrometer Bruker AV III. The chemical shifts are denoted in $\delta$ units (ppm) relative to TMS $(\delta=0.00)$ for protons ${ }^{1} \mathrm{H}$ : s (singlet), t (triplet), and $\mathrm{m}$ (multiplet). Melting points $\left({ }^{\circ} \mathrm{C}\right)$ were measured in open glass capillaries using a Veego (VMP-MP) melting point apparatus and are uncorrected. Infrared spectra $\left(\nu, \mathrm{cm}^{-1}\right)$ were recorded on a Perkin-Elmer spectrophotometer model RX I. Elemental analyses $(\mathrm{C}, \mathrm{H}, \mathrm{N})$ were in full agreement with the proposed structures within $\pm 0.5 \%$ of the theoretical values on a Carlo Erba 1108 analyzer. Monitoring the reactions and checking the purity of the final products were carried out by thin layer chromatography (TLC) using silica gel precoated aluminum sheets (Merck, 60-120 mesh) and visualization with ultraviolet light (UV) at $365 \mathrm{~nm}$ and $254 \mathrm{~nm}$.

4.1.1. Procedure for the Synthesis of 4,6-Diaryl-2-(4-morpholinyl/1-pyrrolidinyl/1-piperidinyl) Pyrimidines. The starting compounds (chalcones) were prepared by the ClaisenSchmidt condensation [16]. A mixture of substituted chalcone $(0.002 \mathrm{~mol}), \mathrm{SBT}(0.0022 \mathrm{~mol})$, heterocyclic secondary amine $(0.0024 \mathrm{~mol})$, and $\mathrm{ZnO}(15 \mathrm{~mol} \%)$ in DMF $(10 \mathrm{~mL})$ was taken in a $100 \mathrm{~mL}$ round bottomed flask and heated at $100^{\circ} \mathrm{C}$ temperature for $6 \mathrm{~h}$ under vigorous stirring. After completion of the reaction as indicated by TLC [Benzene: Ethylacetate, 9:1v/v], reaction mixture was cooled at room temperature and filtered to separate the catalyst. Removal of the excess of solvent under reduced pressure gave crude solid which on recrystallization afforded the title products. 
4.1.2. Spectral Analysis of 4,6-Diaryl-2-(4-morpholinyl)-pyrimidines $3 \boldsymbol{a}-\boldsymbol{g}$. (3a). Yield $86 \%, \mathrm{mp} 105-107^{\circ} \mathrm{C}$. IR (KBr): $v$ 3460, 3115, 1598, 1481, $1253 \mathrm{~cm}^{-1} .{ }^{1} \mathrm{H}$ NMR $(400 \mathrm{MHz}$, $\left.\mathrm{CDCl}_{3}\right): \delta 3.45-3.65\left(\mathrm{~m}, 4 \mathrm{H},-\mathrm{CH}_{2}-\mathrm{N}-\mathrm{CH}_{2}-\right), 3.70-3.90(\mathrm{~m}$, $\left.4 \mathrm{H},-\mathrm{CH}_{2}-\mathrm{O}-\mathrm{CH}_{2}-\right), 6.80-7.30(\mathrm{~m}, 5 \mathrm{H}, \mathrm{Ar}-\mathrm{H}), 7.70(\mathrm{~s}, 1 \mathrm{H})$, 8.48-8.62 (m, 2H), 12.05 (s, 1H, Ar-OH) ppm. ${ }^{13} \mathrm{C}$ NMR $\left(400 \mathrm{MHz}, \mathrm{CDCl}_{3}\right): \delta 47.52,69.51,123.80,126.45,128.65$, $130.23,143.46,148.53,150.50,155.90 \mathrm{ppm}$. MS $\mathrm{m} / z 423$ $\left(\mathrm{M}^{+}\right)$. Anal. calcd for $\mathrm{C}_{20} \mathrm{H}_{17} \mathrm{~N}_{5} \mathrm{O}_{6}$ : C 56.74; $\mathrm{H} 4.05 ; \mathrm{N} 16.54$. Found: C 56.70, H 3.99, N 16.49.

(3b). Yield $90 \%, \mathrm{mp} 165-167^{\circ} \mathrm{C}$. IR (KBr): v 3469, $3125,1600,1478,1252,861 \mathrm{~cm}^{-1}$. ${ }^{1} \mathrm{H}$ NMR $(400 \mathrm{MHz}$, $\left.\mathrm{CDCl}_{3}\right): 3.50-3.70\left(\mathrm{~m}, 4 \mathrm{H},-\mathrm{CH}_{2}-\mathrm{N}-\mathrm{CH}_{2}-\right)$, 3.75-3.90 (m, $\left.4 \mathrm{H},-\mathrm{CH}_{2}-\mathrm{O}-\mathrm{CH}_{2}-\right), 6.80-7.15(\mathrm{~m}, 4 \mathrm{H}, \mathrm{Ar}-\mathrm{H}), 7.80$ (s, $\left.1 \mathrm{H}\right)$, 8.25-8.55 (m, 2H), 12.00 (s, 1H, Ar-OH) ppm. ${ }^{13} \mathrm{C}$ NMR $\left(400 \mathrm{MHz}, \mathrm{CDCl}_{3}\right): \delta 47.45,68.60,123.85,125.80,128.80$ $130.23,142.45,148.50,151.12,155.90,159.53 \mathrm{ppm} \mathrm{MS} \mathrm{m/z}$ $502\left(\mathrm{M}^{+}\right)$. Anal. calcd For $\mathrm{C}_{20} \mathrm{H}_{16} \mathrm{BrN}_{5} \mathrm{O}_{6}$ : C 47.83; H 3.21; N 13.94. Found: C 47.81, H 3.18, N 13.92.

(3c). Yield $89 \%, \mathrm{mp} 100-102^{\circ} \mathrm{C}$. IR (KBr): $v$ 3462, 3116, $1595,1475,1250,863 \mathrm{~cm}^{-1} \cdot{ }^{1} \mathrm{H} \mathrm{NMR}\left(400 \mathrm{MHz}, \mathrm{CDCl}_{3}\right)$ : $\delta$ 3.55-3.70 (m, 4H, $\left.-\mathrm{CH}_{2}-\mathrm{N}-\mathrm{CH}_{2}-\right), 3.60-3.88(\mathrm{~m}, 4 \mathrm{H}$, $\left.-\mathrm{CH}_{2}-\mathrm{O}-\mathrm{CH}_{2}-\right)$, 6.88-7.45 (m, 4H, Ar-H), 7.78 (s, 1H), 8.45-8.63 (m, 2H), 12.10 (s, 1H, Ar-OH) ppm. ${ }^{13} \mathrm{C}$ NMR $\left(400 \mathrm{MHz}, \mathrm{CDCl}_{3}\right): \delta 47.42,68.90,124.81,126.15,127.80$ $131.25,141.15,148.20,152.10,155.35,158.50 \mathrm{ppm}$. MS m/z $502\left(\mathrm{M}^{+}\right)$. Anal. calcd For $\mathrm{C}_{20} \mathrm{H}_{16} \mathrm{BrN}_{5} \mathrm{O}_{6}$ : C 47.83; H 3.21; N 13.94. Found: C 47.85, H 3.17, N 13.90.

(3d). Yield $88 \%, \mathrm{mp} 160-162^{\circ} \mathrm{C}$. IR (KBr): v 3465, 3115, 1598, 1471, 1253, $\left.810 \mathrm{~cm}^{-1} .{ }^{1} \mathrm{H} \mathrm{NMR} \mathrm{(400} \mathrm{MHz,} \mathrm{CDCl}_{3}\right)$ : $\delta$ 3.45-3.75 (m, 4H, $\left.-\mathrm{CH}_{2}-\mathrm{N}-\mathrm{CH}_{2}-\right), 3.65-3.90(\mathrm{~m}, 4 \mathrm{H}$, $\left.-\mathrm{CH}_{2}-\mathrm{O}-\mathrm{CH}_{2}-\right), 6.80-7.20(\mathrm{~m}, 4 \mathrm{H}, \mathrm{Ar}-\mathrm{H}), 7.75(\mathrm{~s}, 1 \mathrm{H})$, 8.40-8.60 (m, 2H), 11.90 (s, 1H, Ar-OH) ppm. ${ }^{13} \mathrm{C}$ NMR $\left(400 \mathrm{MHz}, \mathrm{CDCl}_{3}\right): \delta 47.45,68.60,123.85,125.80,128.80$ $130.23,142.45,148.50,151.12,155.90,159.53 \mathrm{ppm}$. MS m/z $457\left(\mathrm{M}^{+}\right)$. Anal. calcd For $\mathrm{C}_{20} \mathrm{H}_{16} \mathrm{ClN}_{5} \mathrm{O}_{6}$ : C 52.47; $\mathrm{H} 3.52 ; \mathrm{N}$ 15.30. Found: C 52.43, H 3.51, N 15.28.

(3e). Yield $86 \%, \mathrm{mp} 97-99^{\circ} \mathrm{C}$. IR $(\mathrm{KBr}): v 3473$, $3118,1593,1480,1256,821 \mathrm{~cm}^{-1}$. ${ }^{1} \mathrm{H}$ NMR $(400 \mathrm{MHz}$, $\left.\mathrm{CDCl}_{3}\right): \delta 3.50-3.65\left(\mathrm{~m}, 4 \mathrm{H},-\mathrm{CH}_{2}-\mathrm{N}-\mathrm{CH}_{2}-\right), 3.70-3.92$ $\left(\mathrm{m}, 4 \mathrm{H},-\mathrm{CH}_{2}-\mathrm{O}-\mathrm{CH}_{2}-\right), 6.85-7.25(\mathrm{~m}, 4 \mathrm{H}, \mathrm{Ar}-\mathrm{H}), 7.70$ (s, 1H), 8.45-8.62 (m, 2H), $9.80(\mathrm{~s}, 1 \mathrm{H}, \mathrm{Ar}-\mathrm{OH}), 12.00(\mathrm{~s}$, $1 \mathrm{H}, \mathrm{Ar}-\mathrm{OH}) \mathrm{ppm} .{ }^{13} \mathrm{C} \mathrm{NMR}\left(400 \mathrm{MHz}, \mathrm{CDCl}_{3}\right): \delta$ 47.05, $68.15,124.80,125.95,129.15,131.45,144.23,148.15,150.36$, 155.90, $159.95 \mathrm{ppm}$. MS $\mathrm{m} / z 439\left(\mathrm{M}^{+}\right)$. Anal. calcd For $\mathrm{C}_{20} \mathrm{H}_{17} \mathrm{~N}_{5} \mathrm{O}_{7}$ : C 54.67; H 3.90; N 15.94. Found: C 54.55, H 3.81, N 15.78 .

(3f). Yield 89\%, mp $136-138^{\circ} \mathrm{C}$. IR (KBr): $v 3468,3111$, $1605,1479,1258,818 \mathrm{~cm}^{-1} \cdot{ }^{1} \mathrm{H} \mathrm{NMR}\left(400 \mathrm{MHz}, \mathrm{CDCl}_{3}\right)$ : $\delta 3.50-3.78\left(\mathrm{~m}, 4 \mathrm{H},-\mathrm{CH}_{2}-\mathrm{N}-\mathrm{CH}_{2}-\right), 3.70-3.95(\mathrm{~m}, 4 \mathrm{H}$, $\left.-\mathrm{CH}_{2}-\mathrm{O}-\mathrm{CH}_{2}-\right)$, 6.65-7.05 (m, 4H, Ar-H), 7.55 (s, 1H), 8.42-8.61 (m, 2H), 12.10 (s, 1H, Ar-OH) ppm. ${ }^{13} \mathrm{C} \mathrm{NMR}$ $\left(400 \mathrm{MHz}, \mathrm{CDCl}_{3}\right): \delta 47.05,68.95,123.48,126.95,128.35$, $131.55,144.25,148.86,150.85,156.25,159.25 \mathrm{ppm}$. MS m/z $457\left(\mathrm{M}^{+}\right)$. Anal. calcd For $\mathrm{C}_{20} \mathrm{H}_{16} \mathrm{ClN}_{5} \mathrm{O}_{6}$ : C 52.47; $\mathrm{H} 3.52 ; \mathrm{N}$ 15.30. Found: C 52.41, H 3.49, N 15.29.

(3g). Yield 90\%, mp $125-127^{\circ} \mathrm{C}$. IR (KBr): v 3460, 3114, $1600,1472,1258,820 \mathrm{~cm}^{-1} \cdot{ }^{1} \mathrm{H} \mathrm{NMR}\left(400 \mathrm{MHz}, \mathrm{CDCl}_{3}\right)$ : $\delta$ 3.55-3.78 (m, 4H, $\left.-\mathrm{CH}_{2}-\mathrm{N}-\mathrm{CH}_{2}-\right), 3.72-3.95(\mathrm{~m}, 4 \mathrm{H}$,
$\left.-\mathrm{CH}_{2}-\mathrm{O}-\mathrm{CH}_{2}-\right)$, 6.65-7.08 (m, 4H, Ar-H), 7.58 (s, 1H), 8.42-8.61 (m, 2H), $12.08(\mathrm{~s}, 1 \mathrm{H}, \mathrm{Ar}-\mathrm{OH}) \mathrm{ppm} .{ }^{13} \mathrm{C} \mathrm{NMR}$ $\left(400 \mathrm{MHz}, \mathrm{CDCl}_{3}\right): \delta 47.05,68.95,124.48,125.95,127.35$, $131.55,145.25,147.52,150.85,156.65,159.25 \mathrm{ppm}$. MS m/z $457\left(\mathrm{M}^{+}\right)$. Anal. calcd For $\mathrm{C}_{20} \mathrm{H}_{16} \mathrm{ClN}_{5} \mathrm{O}_{6}$ : C 52.47; H 3.52; N 15.30. Found: C 52.40, H 3.45, N 15.23.

4.1.3. Spectral Analysis of 4,6-Diaryl-2-(1-pyrrolidinyl)-pyrimidines) $4 \boldsymbol{a}-\boldsymbol{g}$. (4a). Yield $88 \%, \mathrm{mp} 81-83^{\circ} \mathrm{C}$. IR (KBr): $v$ $3462,3115,1598,1475,1266 \mathrm{~cm}^{-1}$. ${ }^{1} \mathrm{H}$ NMR $(400 \mathrm{MHz}$, $\left.\mathrm{CDCl}_{3}\right): \delta 1.70-1.90\left(\mathrm{~m}, 4 \mathrm{H},-\mathrm{CH}_{2}-\mathrm{CH}_{2}-\right), 3.72-3.90(\mathrm{~m}$, $\left.4 \mathrm{H},-\mathrm{CH}_{2}-\mathrm{N}-\mathrm{CH}_{2}-\right), 6.80-7.20$ (m, 4H, Ar-H), 7.35 (s, 1H), 8.41-8.63 (m, 2H), $11.80(\mathrm{~s}, 1 \mathrm{H}, \mathrm{Ar}-\mathrm{OH}) \mathrm{ppm} .{ }^{13} \mathrm{C} \mathrm{NMR}$ $\left(400 \mathrm{MHz}, \mathrm{CDCl}_{3}\right): \delta$ 25.23, 27.68, 47.80, 123.15, 126.26, $128.30,130.13,143.50,148.45,155.30 \mathrm{ppm}$. MS $\mathrm{m} / z 407$ $\left(\mathrm{M}^{+}\right)$. Anal. calcd for $\mathrm{C}_{20} \mathrm{H}_{17} \mathrm{~N}_{5} \mathrm{O}_{5}$ : C 58.97; $\mathrm{H} 4.21 ; \mathrm{N} 17.19$. Found: C 58.93, H 4.18, N 17.15.

(4b). Yield 89\%, mp 150-152 ${ }^{\circ} \mathrm{C}$. IR (KBr): v 3460, 3112, $1595,1477,1258,868 \mathrm{~cm}^{-1} \cdot{ }^{1} \mathrm{H}$ NMR $\left(400 \mathrm{MHz}, \mathrm{CDCl}_{3}\right): \delta$ 1.70-1.93 (m, 4H, $\left.-\mathrm{CH}_{2}-\mathrm{CH}_{2}-\right)$, 3.61-3.79 (m, 4H, $-\mathrm{CH}_{2}-$ $\left.\mathrm{N}-\mathrm{CH}_{2}-\right)$, 6.90-7.25 (m, 4H, Ar-H), 7.61 (s,1H), 8.41-8.62 $(\mathrm{m}, 2 \mathrm{H}), 12.01$ (s, 1H, Ar-OH) ppm. ${ }^{13} \mathrm{C} \mathrm{NMR}(400 \mathrm{MHz}$, $\left.\mathrm{CDCl}_{3}\right): \delta 25.25,27.96,46.45,124.45,126.85,129.80,130.50$, $142.50,147.22,148.87,155.58, \mathrm{MS} m / z 486\left(\mathrm{M}^{+}\right)$. Anal. calcd For $\mathrm{C}_{20} \mathrm{H}_{16} \mathrm{BrN}_{5} \mathrm{O}_{5}$ : C 49.40; $\mathrm{H}$ 3.32; $\mathrm{N}$ 14.40. Found: $\mathrm{C}$ 49.38, H 3.28, N 14.35.

(4c). Yield $86 \%$, mp $161-162^{\circ} \mathrm{C}$. IR (KBr): $v$ 3468, 3121 , $1603,1478,1265,865 \mathrm{~cm}^{-1} \cdot{ }^{1} \mathrm{H}$ NMR $\left(400 \mathrm{MHz}, \mathrm{CDCl}_{3}\right): \delta$ 1.71-1.98 (m, 4H, $\left.-\mathrm{CH}_{2}-\mathrm{CH}_{2}-\right)$, 3.52-3.75 (m, 4H, $-\mathrm{CH}_{2}-$ $\left.\mathrm{N}-\mathrm{CH}_{2}-\right)$, 6.90-7.35 (m, 4H, Ar-H), 7.70 (s, 1H), 8.40-8.60 $(\mathrm{m}, 2 \mathrm{H}), 11.95$ (s, 1H, Ar-OH) ppm. ${ }^{13} \mathrm{C}$ NMR $(400 \mathrm{MHz}$, $\left.\mathrm{CDCl}_{3}\right): \delta 25.56,27.25,47.00,124.56,126.12,129.11,130.50$, 141.00, 146.00, 148.56, 155.01. ppm MS m/z $486\left(\mathrm{M}^{+}\right)$. Anal. calcd For $\mathrm{C}_{20} \mathrm{H}_{16} \mathrm{BrN}_{5} \mathrm{O}_{5}$ : C 49.40; $\mathrm{H}$ 3.32; N 14.40. Found: C 49.37, H 3.31, N 14.37.

(4d). Yield $86 \%, \mathrm{mp} 175-176^{\circ} \mathrm{C}$. IR (KBr): v 3470, 3120, 1596, 1481, 1261, $815 \mathrm{~cm}^{-1} \cdot{ }^{1} \mathrm{H}$ NMR (400 MHz, $\left.\mathrm{CDCl}_{3}\right): \delta$ 1.70-1.90 (m, 4H, $\left.-\mathrm{CH}_{2}-\mathrm{CH}_{2}-\right)$, 3.52-3.70 (m, 4H, $-\mathrm{CH}_{2}-$ $\mathrm{N}-\mathrm{CH}_{2}-$ ), 7.00-7.35 (m, 4H, Ar-H), 7.70 (s, 1H), 8.35-8.55 $(\mathrm{m}, 2 \mathrm{H}), 12.00$ (s, 1H, Ar-OH) ppm. ${ }^{13} \mathrm{C} \mathrm{NMR}(400 \mathrm{MHz}$, $\left.\mathrm{CDCl}_{3}\right): \delta 25.05,27.89,47.78,125.05,126.56,129.55,130.02$, $140.15,146.85,147.95,155.85,159.36 \mathrm{ppm}$. MS $\mathrm{m} / z 441$ $\left(\mathrm{M}^{+}\right)$. Anal. calcd For $\mathrm{C}_{20} \mathrm{H}_{16} \mathrm{ClN}_{5} \mathrm{O}_{5}$ : C 54.37; $\mathrm{H} 3.65 ; \mathrm{N}$ 15.85. Found: C 54.35, H 3.60, N 15.81.

(4e). Yield $90 \%, \mathrm{mp} 78-80^{\circ} \mathrm{C}$. IR $(\mathrm{KBr}): v 3455,3110$, 1600, 1475, 1258, $818 \mathrm{~cm}^{-1}$. ${ }^{1} \mathrm{H}$ NMR (400 MHz, $\left.\mathrm{CDCl}_{3}\right)$ : $\delta$ 1.70-1.92 (m, 4H, $\left.-\mathrm{CH}_{2}-\mathrm{CH}_{2}-\right), 3.55-3.73(\mathrm{~m}, 4 \mathrm{H},-$ $\left.\mathrm{CH}_{2}-\mathrm{N}-\mathrm{CH}_{2}-\right)$, 6.85-7.25 (m, 4H, Ar-H), $7.75(\mathrm{~s}, 1 \mathrm{H})$, 8.41-8.60 (m, 2H), 9.56 (s, 1H, Ar-OH), 12.01 (s, 1H, Ar$\mathrm{OH})$ ppm. ${ }^{13} \mathrm{C} \mathrm{NMR}\left(400 \mathrm{MHz}, \mathrm{CDCl}_{3}\right): \delta$ 25.05, 27.80, 47.06, 124.00, 126.45, 128.05, 130.54, 139.25, 146.02, 147.30, 156.26, $159.80 \mathrm{ppm}$. MS $\mathrm{m} / z 423\left(\mathrm{M}^{+}\right)$. Anal. calcd For $\mathrm{C}_{20} \mathrm{H}_{17} \mathrm{~N}_{5} \mathrm{O}_{6}$ : C 56.74; $\mathrm{H} 4.05 ; \mathrm{N} 16.54$. Found: C 56.65, H 3.92, N 16.42.

(4f). Yield $88 \%, \mathrm{mp} 125-127^{\circ} \mathrm{C}$. IR $(\mathrm{KBr}): v 3470,3135$, $1615,1469,1245,819 \mathrm{~cm}^{-1} \cdot{ }^{1} \mathrm{H}$ NMR $\left(400 \mathrm{MHz}, \mathrm{CDCl}_{3}\right): \delta$ 1.65-1.88 (m, 4H, $\left.-\mathrm{CH}_{2}-\mathrm{CH}_{2}-\right)$, 3.58-3.78 (m, 4H, $-\mathrm{CH}_{2}-$ $\mathrm{N}-\mathrm{CH}_{2}-$ ), 6.85-7.20 (m, 4H, Ar-H), 7.65 (s, 1H), 8.35-8.55 
(m, 2H), 12.00 (s, 1H, Ar-OH) ppm. ${ }^{13} \mathrm{C}$ NMR $(400 \mathrm{MHz}$, $\left.\mathrm{CDCl}_{3}\right): \delta 25.05,27.80,47.12,124.45,126.85,127.00,129.50$, $141.00,146.01,147.86,156.14,159.15 \mathrm{ppm}$. MS $\mathrm{m} / \mathrm{z} 441$ $\left(\mathrm{M}^{+}\right)$. Anal. calcd For $\mathrm{C}_{20} \mathrm{H}_{16} \mathrm{ClN}_{5} \mathrm{O}_{5}$ : C 54.37; H 3.65; N 15.85. Found: C 54.34, H 3.63, N 15.81.

(4g). Yield 90\%, mp 102-104 ${ }^{\circ} \mathrm{C}$. IR (KBr): $\nu$ 3475, 3131, $1612,1470,1248,822 \mathrm{~cm}^{-1} .{ }^{1} \mathrm{H}$ NMR $\left(400 \mathrm{MHz}, \mathrm{CDCl}_{3}\right): \delta$ 1.68-1.86 (m, $\left.4 \mathrm{H},-\mathrm{CH}_{2}-\mathrm{CH}_{2}-\right), 3.58-3.80\left(\mathrm{~m}, 4 \mathrm{H},-\mathrm{CH}_{2}-\right.$ $\left.\mathrm{N}-\mathrm{CH}_{2}-\right), 6.82-7.20(\mathrm{~m}, 4 \mathrm{H}, \mathrm{Ar}-\mathrm{H}), 7.65(\mathrm{~s}, 1 \mathrm{H}), 8.33-8.52$ $(\mathrm{m}, 2 \mathrm{H}), 12.02$ (s, $1 \mathrm{H}, \mathrm{Ar}-\mathrm{OH}) \mathrm{ppm} .{ }^{13} \mathrm{C}$ NMR $(400 \mathrm{MHz}$, $\left.\mathrm{CDCl}_{3}\right): \delta 25.08,27.82,47.12,123.45,125.80,127.05,129.55$, $141.06,147.05,147.86,156.20,159.10 \mathrm{ppm}$. MS m/z 441 $\left(\mathrm{M}^{+}\right)$. Anal. calcd For $\mathrm{C}_{20} \mathrm{H}_{16} \mathrm{ClN}_{5} \mathrm{O}_{5}$ : C 54.37; $\mathrm{H}$ 3.65; N 15.85. Found: C 54.30, H 3.60, N 15.80.

4.1.4. Spectral Analysis of 4,6-Diaryl-2-(1-piperidinyl)-pyrimidines) $5 \mathbf{a}-\mathbf{g}$. (5a). Yield $89 \%, \mathrm{mp} 116-118^{\circ} \mathrm{C}$. IR (KBr): $v$ 3460, 3119, 1599, 1479, $1259 \mathrm{~cm}^{-1} .{ }^{1} \mathrm{H}$ NMR $(400 \mathrm{MHz}$, $\left.\mathrm{CDCl}_{3}\right): \delta 1.40-1.62\left(\mathrm{~m}, 6 \mathrm{H},-\left(\mathrm{CH}_{2}\right)_{2}-\right), 3.40-3.71(\mathrm{~m}$, $\left.4 \mathrm{H},-\mathrm{CH}_{2}-\mathrm{N}-\mathrm{CH}_{2}-\right), 3.79$ (s, 3H, Ar- $\left.\mathrm{OCH}_{3}\right), 6.90-7.20$ $(\mathrm{m}, 4 \mathrm{H}, \mathrm{Ar}-\mathrm{H}), 7.65(\mathrm{~s}, 1 \mathrm{H}), 8.35-8.60(\mathrm{~m}, 2 \mathrm{H}), 11.25(\mathrm{~s}$, $1 \mathrm{H}, \mathrm{Ar}-\mathrm{OH}) \mathrm{ppm} .{ }^{13} \mathrm{C}$ NMR $\left(400 \mathrm{MHz} \mathrm{CDCl}_{3}\right): \delta 25.20$, $27.35,47.55,123.75,128.54,130.55,143.85,148.75,150.45$, 155.90 ppm. MS m/z $421\left(\mathrm{M}^{+}\right)$. Anal. calcd for $\mathrm{C}_{21} \mathrm{H}_{19} \mathrm{~N}_{5} \mathrm{O}_{5}$ : C 59.84; H 4.54; N 16.62. Found: C 59.80, H 4.49, N 16.68.

(5b). Yield $86 \%, \mathrm{mp} 171-173^{\circ} \mathrm{C}$. IR ( $\left.\mathrm{KBr}\right): v 3465,3111$, $1605,1481,1261,855 \mathrm{~cm}^{-1} .{ }^{1} \mathrm{H}$ NMR $\left(400 \mathrm{MHz}, \mathrm{CDCl}_{3}\right)$ : $\delta 1.30-1.58\left(\mathrm{~m}, 6 \mathrm{H},-\left(\mathrm{CH}_{2}\right)_{3}-\right), 3.45-3.72\left(\mathrm{~m}, 4 \mathrm{H},-\mathrm{CH}_{2}-\right.$ $\left.\mathrm{N}-\mathrm{CH}_{2}-\right), 6.60-7.00$ (m, 4H, Ar-H), 7.65 (s, 1H), 8.30-8.55 (m, 2H), 11.90 (s, 1H, Ar-OH) ppm. ${ }^{13} \mathrm{C}$ NMR $(400 \mathrm{MHz}$, $\left.\mathrm{CDCl}_{3}\right): \delta 25.42,27.40,47.75,124.00,129.15,130.05,143.15$, $148.00,148.85,150.04,155.00 \mathrm{ppm}$. MS $\mathrm{m} / z 400\left(\mathrm{M}^{+}\right)$. Anal. calcd For $\mathrm{C}_{21} \mathrm{H}_{18} \mathrm{BrN}_{5} \mathrm{O}_{5}$ : C 50.41; $\mathrm{H} 3.63 ; \mathrm{N}$ 14.00. Found: C 50.38, H 3.61, N 13.98 .

(5c). Yield 89\%, mp $152-1154^{\circ} \mathrm{C}$. IR ( $\left.\mathrm{KBr}\right): \nu 3460,3115$, 1610, 1490, 1255, $861 \mathrm{~cm}^{-1} .{ }^{1} \mathrm{H}$ NMR $\left(400 \mathrm{MHz}, \mathrm{CDCl}_{3}\right)$ : $\delta$ 1.30-1.50 (m, 6H, $\left.-\left(\mathrm{CH}_{2}\right)_{2}-\right), 4.40-4.71\left(\mathrm{~m}, 4 \mathrm{H},-\mathrm{CH}_{2}-\right.$ $\left.\mathrm{N}-\mathrm{CH}_{2}-\right), 6.90-7.35$ (m, 4H, Ar-H), $7.70(\mathrm{~s}, 1 \mathrm{H}), 8.45-8.62$ $(\mathrm{m}, 2 \mathrm{H}), 11.65(\mathrm{~s}, 1 \mathrm{H}, \mathrm{Ar}-\mathrm{OH}) \mathrm{ppm} .{ }^{13} \mathrm{C}$ NMR $(400 \mathrm{MHz}$, $\left.\mathrm{CDCl}_{3}\right): \delta 25.45,27.40,47.80,124.65,129.00,129.95,143.58$, $147.05,148.95,151.15,155.65 \mathrm{ppm}$. MS $m / z 400\left(\mathrm{M}^{+}\right)$. Anal. calcd For $\mathrm{C}_{21} \mathrm{H}_{18} \mathrm{BrN}_{5} \mathrm{O}_{5}$ : C 50.41; $\mathrm{H} 3.63 ; \mathrm{N}$ 14.00. Found: C 50.37, H 3.65, N 14.02.

(5d). Yield $87 \%, \mathrm{mp} 201-203^{\circ} \mathrm{C}$. IR ( $\left.\mathrm{KBr}\right): \nu 3469,3118$, $1603,1479,1253,802 \mathrm{~cm}^{-1} .{ }^{1} \mathrm{H}$ NMR $\left(400 \mathrm{MHz}, \mathrm{CDCl}_{3}\right)$ : $1.40-1.65\left(\mathrm{~m}, 6 \mathrm{H},-\left(\mathrm{CH}_{2}\right)_{2}-\right), 3.42-3.65\left(\mathrm{~m}, 4 \mathrm{H},-\mathrm{CH}_{2}-\mathrm{N}-\right.$ $\left.\mathrm{CH}_{2}-\right)$, 6.95-7.35 (m, 4H, Ar-H), $7.66(\mathrm{~s}, 1 \mathrm{H}), 8.42-8.65$ $(\mathrm{m}, 2 \mathrm{H}), 12.00$ (s, $1 \mathrm{H}, \mathrm{Ar}-\mathrm{OH}) \mathrm{ppm} .{ }^{13} \mathrm{C}$ NMR $(400 \mathrm{MHz}$, $\left.\mathrm{CDCl}_{3}\right): \delta 25.55,27.05,47.80,124.15,129.45,130.02,143.15$, $147.00,148.23,151.01,156.35 \mathrm{ppm}$. MS $\mathrm{m} / z 455\left(\mathrm{M}^{+}\right)$. Anal Anal. calcd For $\mathrm{C}_{21} \mathrm{H}_{18} \mathrm{ClN}_{5} \mathrm{O}_{5}$ : C 55.33; H 3.98; N 15.36. Found: C 55.31, H 3.95, N 15.33.

(5e). Yield 87\%, mp 100-102 ${ }^{\circ} \mathrm{C}$. IR (KBr): $v 3468,3121$, 1596, 1471, 1261, $820 \mathrm{~cm}^{-1} .{ }^{1} \mathrm{H}$ NMR $\left(400 \mathrm{MHz}, \mathrm{CDCl}_{3}\right)$ : $\delta 1.48-1.68\left(\mathrm{~m}, 6 \mathrm{H},-\left(\mathrm{CH}_{2}\right)_{3}-\right), 3.48-3.71\left(\mathrm{~m}, 4 \mathrm{H},-\mathrm{CH}_{2}-\right.$ $\left.\mathrm{N}-\mathrm{CH}_{2}-\right), 6.92-7.25(\mathrm{~m}, 4 \mathrm{H}, \mathrm{Ar}-\mathrm{H}), 7.65(\mathrm{~s}, 1 \mathrm{H}), 8.42-$ $8.60(\mathrm{~m}, 2 \mathrm{H}), 8.56(\mathrm{~s}, 1 \mathrm{H}, \mathrm{Ar}-\mathrm{OH}), 12.10(\mathrm{~s}, 1 \mathrm{H}, \mathrm{Ar}-$ $\mathrm{OH}) \mathrm{ppm} .{ }^{13} \mathrm{C} \mathrm{NMR}\left(400 \mathrm{MHz}, \mathrm{CDCl}_{3}\right): \delta 25.50,27.23$,
47.56, 124.15, 130.01, 131.55, 143.50, 147.48, 148.01, 150.45, $156.85,159.50 \mathrm{ppm}$. MS $\mathrm{m} / \mathrm{z} 437\left(\mathrm{M}^{+}\right)$. Anal. calcd For $\mathrm{C}_{21} \mathrm{H}_{19} \mathrm{~N}_{5} \mathrm{O}_{6}$ : C 57.66; $\mathrm{H} 4.38 ; \mathrm{N}$ 16.01. Found: C 57.51, $\mathrm{H}$ 4.25, N 15.92.

(5f). Yield 86\%, mp $201-203^{\circ} \mathrm{C}$. IR (KBr): $v$ 3464, 3118, $1601,1468,1258,809 \mathrm{~cm}^{-1} .{ }^{1} \mathrm{H}$ NMR $\left(400 \mathrm{MHz}, \mathrm{CDCl}_{3}\right)$ : $\delta 1.35-1.53\left(\mathrm{~m}, 6 \mathrm{H},-\left(\mathrm{CH}_{2}\right)_{3}-\right), 3.48-3.70\left(\mathrm{~m}, 4 \mathrm{H},-\mathrm{CH}_{2}-\right.$ $\left.\mathrm{N}-\mathrm{CH}_{2}-\right), 6.80-7.30(\mathrm{~m}, 4 \mathrm{H}, \mathrm{Ar}-\mathrm{H}), 7.60(\mathrm{~s}, 1 \mathrm{H}), 8.35-8.58$ $(\mathrm{m}, 2 \mathrm{H}), 12.00$ (s, $1 \mathrm{H}, \mathrm{Ar}-\mathrm{OH}) \mathrm{ppm} .{ }^{13} \mathrm{C}$ NMR $(400 \mathrm{MHz}$, $\left.\mathrm{CDCl}_{3}\right): \delta 25.21,27.23,47.15,124.56,130.00,131.35,141.52$, $146.14,148.45,151.56,156.42,159.23 \mathrm{ppm}$. MS m/z 455 $\left(\mathrm{M}^{+}\right)$. Anal. calcd For $\mathrm{C}_{21} \mathrm{H}_{18} \mathrm{ClN}_{5} \mathrm{O}_{5}$ : C 55.33; $\mathrm{H} 3.98 ; \mathrm{N}$ 15.36. Found: C 55.31, H 3.95, N 15.30.

(5g). Yield $88 \%$, mp $160-162^{\circ} \mathrm{C}$. IR (KBr): $v 3466,3120$, 16012, 1465, 1258, $810 \mathrm{~cm}^{-1} \cdot{ }^{1} \mathrm{H}$ NMR $\left(400 \mathrm{MHz}, \mathrm{CDCl}_{3}\right)$ : $\delta 1.35-1.55\left(\mathrm{~m}, 6 \mathrm{H},-\left(\mathrm{CH}_{2}\right)_{3}-\right), 3.45-3.71\left(\mathrm{~m}, 4 \mathrm{H},-\mathrm{CH}_{2}-\right.$ $\left.\mathrm{N}-\mathrm{CH}_{2}-\right), 6.78-7.32(\mathrm{~m}, 4 \mathrm{H}, \mathrm{Ar}-\mathrm{H}), 7.65(\mathrm{~s}, 1 \mathrm{H}), 8.35-8.58$ $(\mathrm{m}, 2 \mathrm{H}), 12.02$ (s, $1 \mathrm{H}, \mathrm{Ar}-\mathrm{OH}) \mathrm{ppm} .{ }^{13} \mathrm{C}$ NMR $(400 \mathrm{MHz}$, $\left.\mathrm{CDCl}_{3}\right): \delta 25.21,27.25,47.20,123.50,131.02,132.30,140.51$, $145.18,147.41,150.55,157.40,160.20 \mathrm{ppm}$. MS m/z 455 $\left(\mathrm{M}^{+}\right)$. Anal. calcd For $\mathrm{C}_{21} \mathrm{H}_{18} \mathrm{ClN}_{5} \mathrm{O}_{5}$ : C 55.33; H 3.98; N 15.36. Found: C 55.28, H 3.92, N 15.25.

\section{Acknowledgments}

The authors are thankful to Professor B. L. Verma, retired. Professor of Chemistry, M. L. S. University, Udaipur, and Dr. S. Jakhoria, Dean, FASC, MITS University for constant encouragement during this work. They are also thankful to the Head of SAIF, Panjab University, Chandigarh, Punjab, for spectral analysis.

\section{References}

[1] A. Domling and I. Ugi, "Multicomponent reactions with isocyanides," Angewandte Chemie International Edition, vol. 39, pp. 3168-3210, 2000.

[2] L. Yu, B. Chen, and X. Huang, "Multicomponent reactions of allenes, diaryl diselenides, and nucleophiles in the presence of iodosobenzene diacetate: direct synthesis of 3-functionalized2-arylselenyl substituted allyl derivatives," Tetrahedron Letters, vol. 48, no. 6, pp. 925-927, 2007.

[3] J. Zhu and H. Bienayme, Multicomponent Reactions Wiley$V C H, 2005$.

[4] R. A. Sheldon and J. Dakka, "Heterogeneous catalytic oxidations in the manufacture of fine chemicals," Catalysis Today, vol. 19, no. 2, pp. 215-246, 1994.

[5] R. A. Sheldon and R. S. Downing, "Heterogeneous catalytic transformations for environmentally friendly production," Applied Catalysis A, vol. 189, no. 2, pp. 163-183, 1999.

[6] K. Tanabe, Solid Acids and Bases, Academic Press, New York, NY, USA, 1970.

[7] J. C. Trivedi, J. B. Bariwal, K. D. Upadhyay et al., "Improved and rapid synthesis of new coumarinyl chalcone derivatives and their antiviral activity," Tetrahedron Letters, vol. 48, no. 48, pp. 8472-8474, 2007.

[8] M. Chen, S. B. Christensen, L. Zhai et al., "The novel oxygenated chalcone, 2,4-dimethoxy-4'-butoxychalcone, exhibits potent activity against human malaria parasite Plasmodium falciparum in vitro and rodent parasites Plasmodium berghei 
and Plasmodium yoelii in vivo," Journal of Infectious Diseases, vol. 176, no. 5, pp. 1327-1333, 1997.

[9] T. Narender, K. Shweta, R. M. Tanvir, S. K. Srinivasa, and S. K. Puri, "Prenylated chalcones isolated from Crotalaria genus inhibits in vitro growth of the human malaria parasite Plasmodium falciparum," Bioorganic and Medicinal Chemistry Letters, vol. 15, no. 10, pp. 2453-2455, 2005.

[10] X. Wu, P. Wilairat, and M. L. Go, "Antimalarial activity of ferrocenyl chalcones," Bioorganic and Medicinal Chemistry Letters, vol. 12, no. 17, pp. 2299-2302, 2002.

[11] M. Liu, P. Wilairat, S. L. Croft, A. L. C. Tan, and M. L. Go, "Structure-activity relationships of antileishmanial and antimalarial chalcones," Bioorganic and Medicinal Chemistry, vol. 11, no. 13, pp. 2729-2738, 2003.

[12] S. F. Nielsen, S. B. Christensen, G. Cruciani, A. Kharazmi, and T. Liljefors, "Antileishmaniai chalcones: statistical design, synthesis, and three- dimensional quantitative structureactivity relationship analysis," Journal of Medicinal Chemistry, vol. 41, no. 24, pp. 4819-4832, 1998.

[13] S. A. Indyah, H. Timmerman, M. Samhoedi, S. Sastrohamidjojo, and H. Van Der Goot, "Synthesis of benzylideneacetophenones and their inhibition of lipid peroxidation," European Journal of Medicinal Chemistry, vol. 35, no. 4, pp. 449-457, 2000.

[14] C. L. Ye, J. W. Liu, D. Z. Wei, Y. H. Lu, and F. Qian, "In vitro anti-tumor activity of $2^{\prime}, 4^{\prime}$-dihydroxy- $6^{\prime}$ - methoxy$3^{\prime}, 5^{\prime}$-dimethylchalcone against six established human cancer cell lines," Pharmacological Research, vol. 50, no. 5, pp. 505510, 2004.

[15] K. L. Ameta, N. S. Rathore, and B. Kumar, "Synthesis and in vitro anti breast cancer activity of some novel 1, 5-benzothiazepine derivatives," Journal of Serbian Chemical Society, vol. 77, no. 6, pp. 725-731, 2012.

[16] K. L. Ameta, B. Kumar, and N. S. Rathore, "Microwave induced improved synthesis of some novel substituted 1, 3diarylpropenones and their antimicrobial activity," E-Journal of Chemistry, vol. 8, no. 2, pp. 665-671, 2011.

[17] M. L. Irene, "Pyrimidine as constituent of natural biologically active compounds," Chemistry and Biodiversity, vol. 2, no. 1, pp. 1-50, 2005.

[18] T. A. Naik and K. H. Chikhalia, "Studies on synthesis of pyrimidine derivatives and their pharmacological evaluation," European Journal of Chemistry, vol. 4, no. 1, pp. 60-66, 2007.

[19] K. L. Ameta, N. S. Rathore, and B. Kumar, "Synthesis of some novel chalcones and their facile one-pot conversion to 2-aminobenzene-1, 3-dicarbonitriles using malononitrile," Analele Universitatii Bucuresti Chimie, vol. 20, no. 1, pp. 15-24, 2011.

[20] P. Sharma, K. F. Hussain, S. Sukhwal, S. Kothari, M. Singhal, and B. L. Verma, "A convenient one-pot synthesis of 2substituted-4,6-diaryl pyrimidines," Indian Journal of Chemistry B, vol. 38, no. 8, pp. 966-968, 1999.

[21] S. Kothari, R. Vyas, and B. L. Verma, "A facile one pot conversion of $3^{\prime}, 5^{\prime}$-dibromo- $4^{\prime}$-hydroxy substituted chalcones to pyrimidine derivatives and their antibacterial and herbicidal activity," Indian Journal of Heterocyclic Chemistry, vol. 8, no. 4, pp. 285-288, 1999.

[22] U. S. Gahlot, S. S. Rao, S. S. Dulawat, K. L. Ameta, and B. L. Verma, "A facile one-pot microwave assisted conversion of $3^{\prime}-5^{\prime}$ - dibromo/diiodo-4' -hydroxy substituted chalcones to 2substituted-4,6- diaryl pyrimidines using S-benzylisothiour- onium chloride (SBT) and their antibacterial activities," Afinidad, vol. 60, no. 508, pp. 558-562, 2003.

[23] M. Kidwai, S. Rastogi, and S. Saxena, "Base catalysed pyrimidine synthesisusing microwave," Bulletin of the Korean Chemical Society, vol. 24, no. 11, pp. 1575-1578, 2003.

[24] P. Shiv, M. V. S. Sharma, A. K. Suryanarayana, A. S. Nigam, A. S. Chauhan, and L. N. S. Tomar, "[PANI/ZnO] composite: catalyst for solvent-free selective oxidation of sulfides," Catalysis Communications, vol. 10, no. 6, pp. 905-912, 2009.

[25] K. L. Ameta, B. Kumar, and N. S. Rathore, "Facile synthesis of some novel 2-substituted-4, 6-diarylpyrimidines using 4'-hydroxy-3', 5' -dinitrochalcones and S-benzylthiouronium chloride," Organic Communication, vol. 5, pp. 1-11, 2012. 


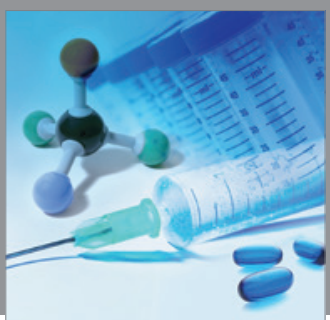

International Journal of

Medicinal Chemistry

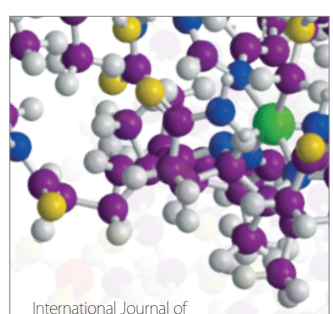

Carbohydrate Chemistry

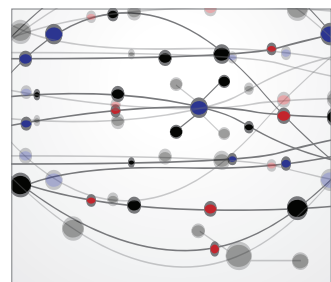

The Scientific World Journal
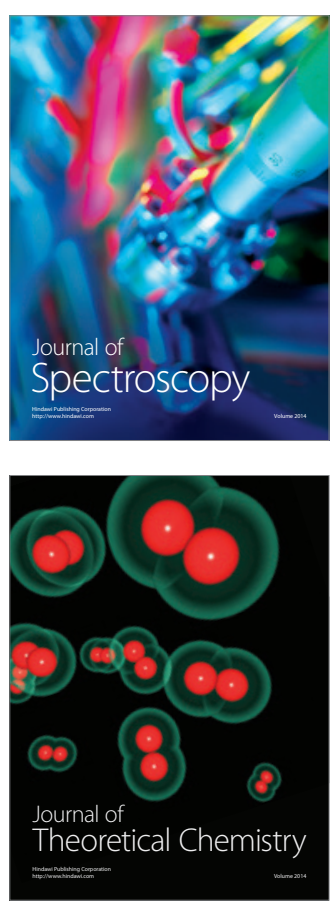
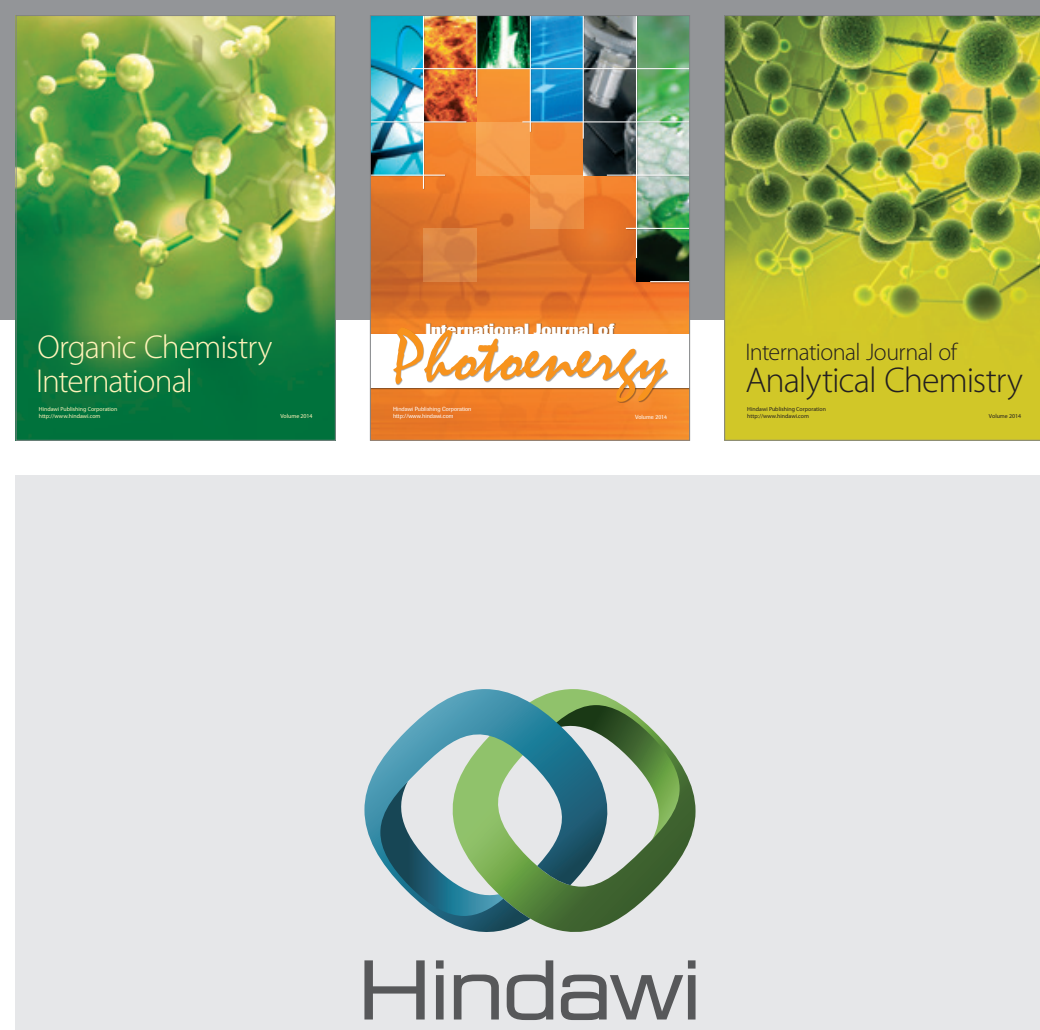

Submit your manuscripts at

http://www.hindawi.com
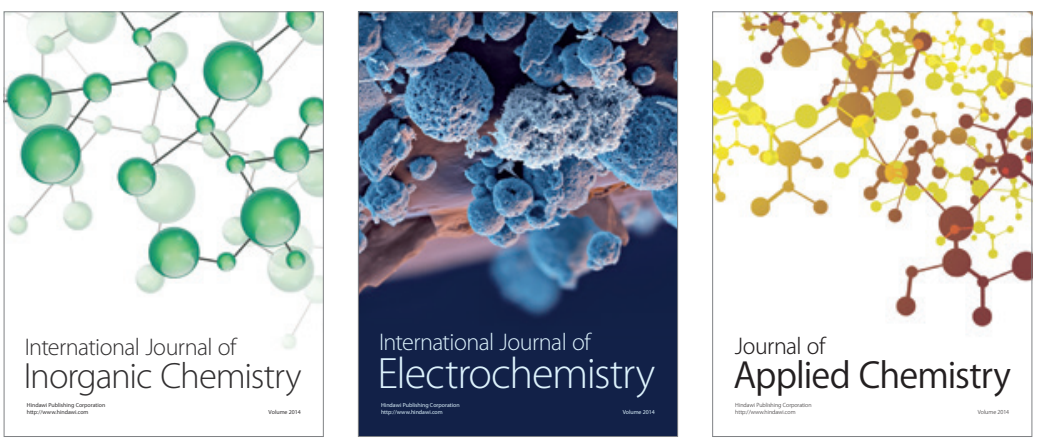

Journal of

Applied Chemistry
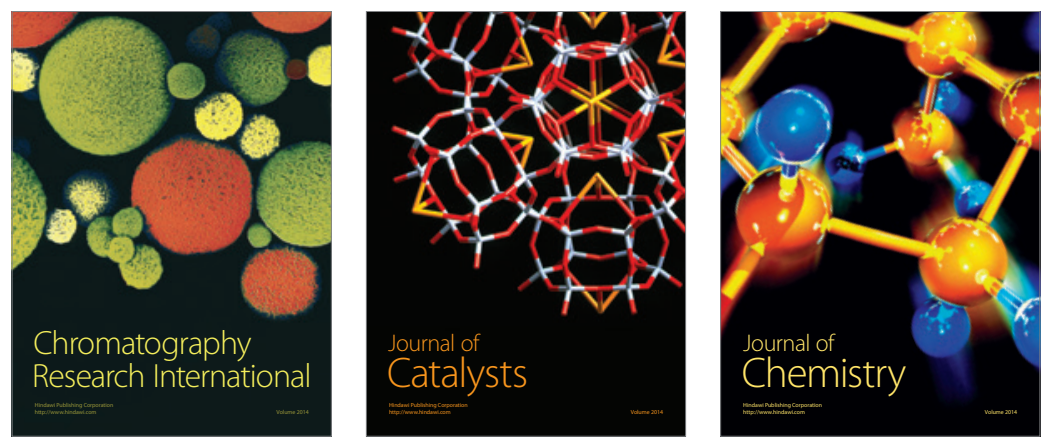
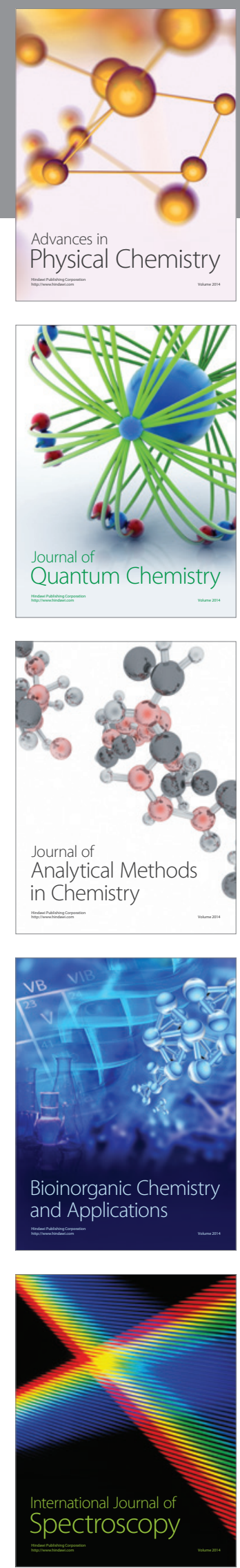